\title{
Ability of ergonomic timeout to reduce musculoskeletal discomfort related to fluoroscopic endoscopy
}

\section{다)(요 $\odot$}

\author{
Authors \\ Yasuki Hori ${ }^{1}$, Taisei Nagai ${ }^{2}$, Kazuki Hayashi ${ }^{1}$, Hiroyuki Izumi ${ }^{3}$, Kiyoko Yokoyama $^{4}$, Takeshi Ebara ${ }^{2}$
}

Institutions

1 Department of Gastroenterology and Metabolism, Nagoya City University Graduate School of Medical Sciences, Nagoya, Japan

2 Department of Occupational and Environmental Health, Nagoya City University Graduate School of Medical Sciences, Nagoya, Japan

3 Department of Ergonomics, Institute of Industrial Ecological Sciences, University of Occupational and Environmental Health, Kitakyushu, Japan

4 Nagoya City University Graduate School of Design and Architecture, Nagoya, Japan

submitted 12.5.2021

accepted after revision $\quad 10.8 .2021$

Bibliography

Endosc Int Open 2021; 09: E1909-E1913

DOI 10.1055/a-1594-2037

ISSN 2364-3722

(c) 2021. The Author(s).

This is an open access article published by Thieme under the terms of the Creative Commons Attribution-NonDerivative-NonCommercial License, permitting copying and reproduction so long as the original work is given appropriate credit. Contents may not be used for commercial purposes, or adapted, remixed, transformed or built upon. (https://creativecommons.org/licenses/by-nc-nd/4.0/)

Georg Thieme Verlag KG, Rüdigerstraße 14,

70469 Stuttgart, Germany
Corresponding author

Takeshi Ebara, PhD, CPE, Department of Occupational and

Environmental Health, Nagoya City University Graduate

School of Medical Sciences, 1 Kawasumi, Mizuho-cho,

Mizuho-ku Nagoya 467-8601, Japan

Fax: +81-52-859-1228.

ebara@med.nagoya-cu.ac.jp

\section{ABSTRACT}

Endoscopists performing fluoroscopy procedures often suffer from musculoskeletal disorders (MSDs) due to persistent static muscle contraction and the load imposed by radiation personal protective equipment. We devised the "practical load-on-the-shoulders releasing technique" (PoRT), integrated it within the work-rest schedule, and investigated its ability to reduce muscular discomfort. The results showed that PoRT lowered discomfort, including by maintaining the head angle closer to the neutral position and keeping the trunk in a stable state, which together resulted in an ideal posture. As a simple, no-cost intervention based on an ergonomic approach, PoRT may contribute to reducing MSDs.

\section{Introduction}

Little is known about musculoskeletal disorders (MSDs) among endoscopists performing fluoroscopic procedures. Fluoroscopy-related MSDs can result from persistent static muscle contraction due to the constrained work conditions. Radiation personal protective equipment (RPPE), including lead aprons, thyroid collars, and lead glasses, imposes an additional load. Some reports have described high MSD levels in endoscopists performing fluoroscopic procedures $[1,2]$, but the physical workloads have seldom been quantified. To prevent MSD, it is essential to optimize the monitor size, height, and distance [3,4] and the height of the treatment bed [4]. However, the introduction of new hardware configurations is not feasible because of high cost. We devised a simple, no-cost countermeasure (the "practical load-on-the-shoulders releasing technique" [PoRT]; the details are given later) based on intermittent work-rest periods during fluoroscopic endoscopic treatment. The aim of this study was to investigate whether PoRT reduces muscular discomfort in endoscopists and to identify the postural features that may contribute to MSDs during endoscopic procedures.

\section{Methods}

Muscular discomfort involving the neck/shoulders, lower back, and lower limbs was assessed by 25 repeated visual analogue scale (VAS) measurements taken at four time points during work days $(9: 00,12: 00,15: 00,18: 00)$ from June 25, 2020 through October 15, 2020. Tuesday and Thursday are the pro- 


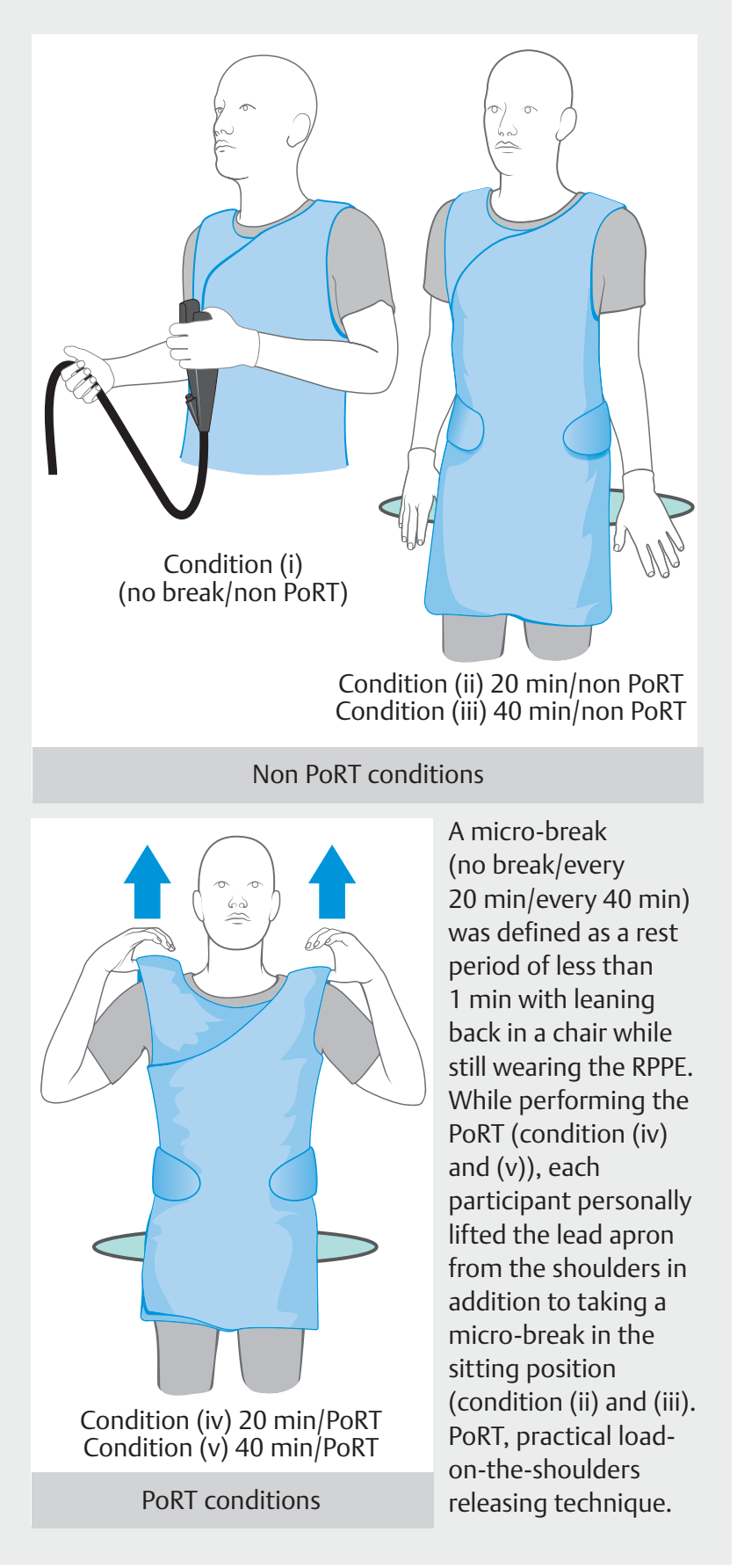

Fig. 1 The five experimental conditions.

cedural days in our institution. Therefore, we collected extensive information on both days. A senior endoscopist (a 41year-old man with no comorbidities) was studied. His body mass index was $20.0 \mathrm{~kg} / \mathrm{m}^{2}$ and he exercised twice weekly. We studied five conditions, as follows: (1) no breaks; (2) microbreak every 20 minutes; (3) micro-break every 40 minutes; (4) micro-break every 20 minutes with PoRT; and (5) micro-break every 40 minutes with PoRT. A micro-break was defined as less than 1 minute of rest ( $\mathbf{F i g . 1}$ ). During the fluoroscopic procedures, operator pitch (the angle when bending forward or backward) and roll (the lateral angle) were measured using a

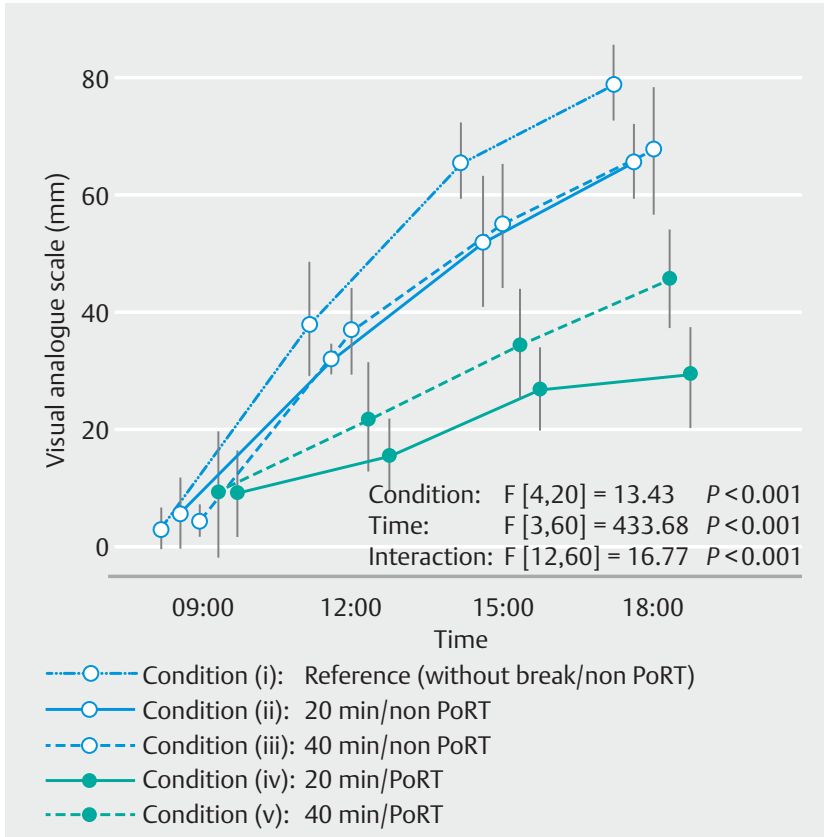

Error bars represent standard deviation.

The vertical bars between pairs represent the significant difference of multiple comparisons at 18:00 $(P<0.01)$

- Fig. 2 Subjective muscular discomfort in the neck/shoulders under the PoRT and non-PoRT conditions during fluoroscopy procedures. There was a main effect of time on muscular discomfort $(F[3,60]=433.68, P<0.001)$. Discomfort was lower in the PoRT compared to non-PoRT condition $(P<0.001)$. The condition $\times$ time interaction effect was significant $(F[12,60]=16.77, P<0.001)$.

wearable accelerometer that estimated head/neck and trunk posture in the sagittal and frontal planes. The study was approved by the Institutional Review Board of Nagoya City University (approval no. 46-20-0003).

\section{Muscular discomfort evaluation}

VAS was used to evaluate muscle fatigue at four time points during the day. Therefore, the participant wore the RPPE from 9:00 to 18:00 (thus, for the entire workday) and was asked to indicate pain in his neck/shoulders, lower back, and lower limbs by marking horizontal lines on a 100-mm VAS (no pain, $0 \mathrm{~mm}$; the most severe pain imaginable, $100 \mathrm{~mm}$ ).

\section{Intervention details}

The five conditions mentioned above were assessed. A microbreak was defined as a rest period of less than 1 minute while still wearing the RPPE. While performing the PoRT, the participant personally lifted the lead apron from his shoulders while taking a micro-break in the sitting position. Working conditions were recorded throughout the entire observational period.

\section{Measurement of head and trunk angles}

Head and trunk angles were determined using the wearable accelerometers. Head angles were estimated with the aid of JINS MEME motion-sensing eyewear (JINS, Tokyo, Japan); this meas- 

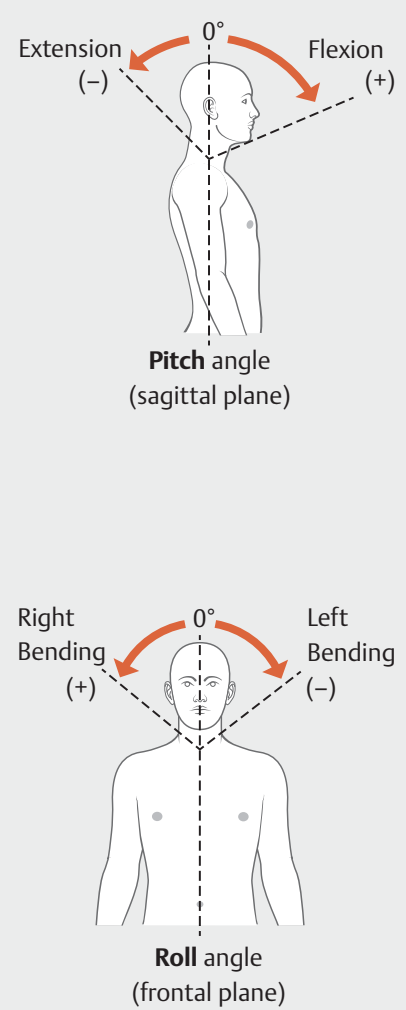
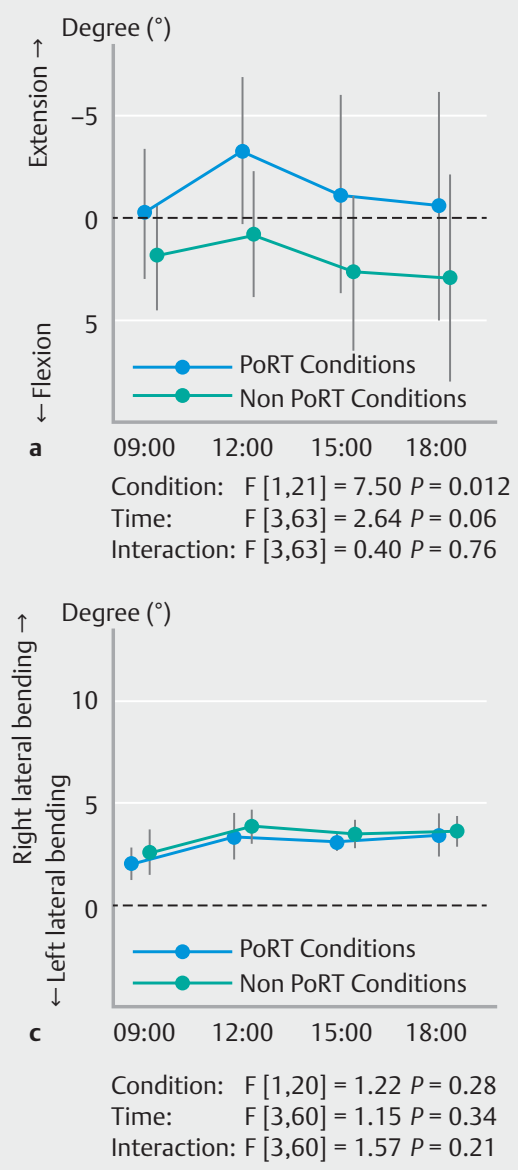
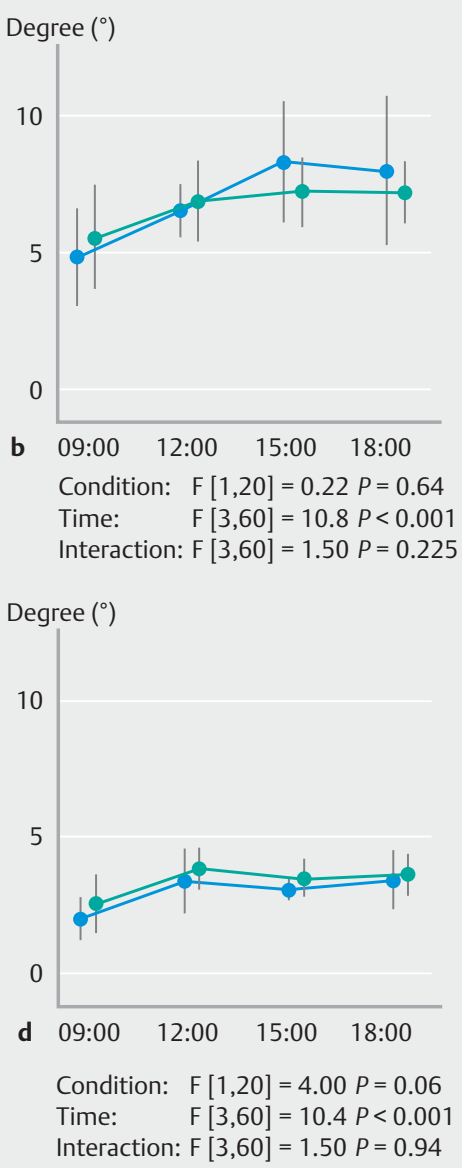

- Fig. 3 Head flexion angle: difference between the PoRT and non-PoRT conditions during fluoroscopy procedures. a, c Mean angle.

b, d Mean displacement. Error bars represent standard deviation.

ures head motion in three dimensions. The pitch (head angle when bending forward or backward) and roll (head angle when bending laterally) were recorded. The front-facing standing position was defined as $0^{\circ}$ for each axis. Trunk angles were estimated using a device worn at the waist (Sensingwear SS-05; Nikon, Tokyo, Japan). The device was used to measure pitch in the sagittal plane and roll in the frontal plane. Both motion-sensing devices yield time-series data at $20 \mathrm{~Hz}$. We recorded the times of all endoscopic procedures and extracted the results.

\section{Sample size and statistical analysis}

The sample size was determined based on a previous study [5]. Given the effect sizes of the conditions (five levels, $f=0.25$ ) and times (four levels, $f=0.4$ ), and assuming $\alpha=0.05$, we estimated that a statistical power $(1-\beta)$ of at least $80 \%$ could be achieved by including five samples in each cell ( 25 repeated measurements under the five conditions). We thus performed two-way repeated-measures analysis of variance with condition and time as the independent variables. Data processing and analyses were conducted with the aid of $\mathrm{R}$ software (version 3.6.3; $\mathrm{R}$ Foundation for Statistical Computing, Vienna, Austria).

\section{Results}

The median number of daily endoscopic procedures was eight (range 5-13). Muscular discomfort in the neck/shoulders increased over time $(F[3,60]=433.68, P<0.001)$ but at 18:00 there was less discomfort with than without PoRT $(P<0.01)$. The interaction between condition and time was significant ( $F$ $[12,60]=16.77, P<0.001$ ) ( Fig.2). Similar muscular discomfort trends were observed for the lower back (condition: $F$ $[4,20]=21.8, P<0.001$, time: $F[3,60]=365.4, P<0.001$, condition $\times$ time interaction: $F[12,60]=14.4, P<0.001)$ and lower limbs (condition: $F[4,20]=12.15, P<0.001$, time: $F[3,60]=$ 275.5, $P<0.001$, interaction: $F[12,60]=11.8, P<0.001)$.

The head flexion angle was significantly closer to the neutral position under the PoRT than the non-PoRT condition $(P=$ 0.012, Fig.3a). Displacement of the head pitch/roll angles, defined as the mean square displacement and based on the average angular change between angle (t+1) and angle $(t)$, increased significantly with time under both the PoRT and nonPoRT conditions (both $P<0.001,>$ Fig. 3b, $>$ Fig. $3 d$ ). The differences between the two conditions with respect to head roll angle ( $\triangleright$ Fig. $\mathbf{3 c}$ ) and the mean trunk pitch/roll angles were not significant ( $\triangleright$ Fig.4a, $\triangleright$ Fig.4c). However, the trunk roll in the 

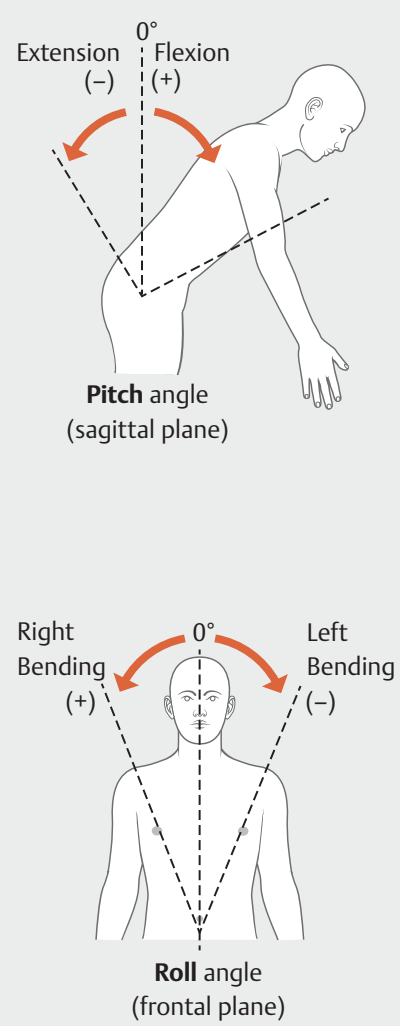
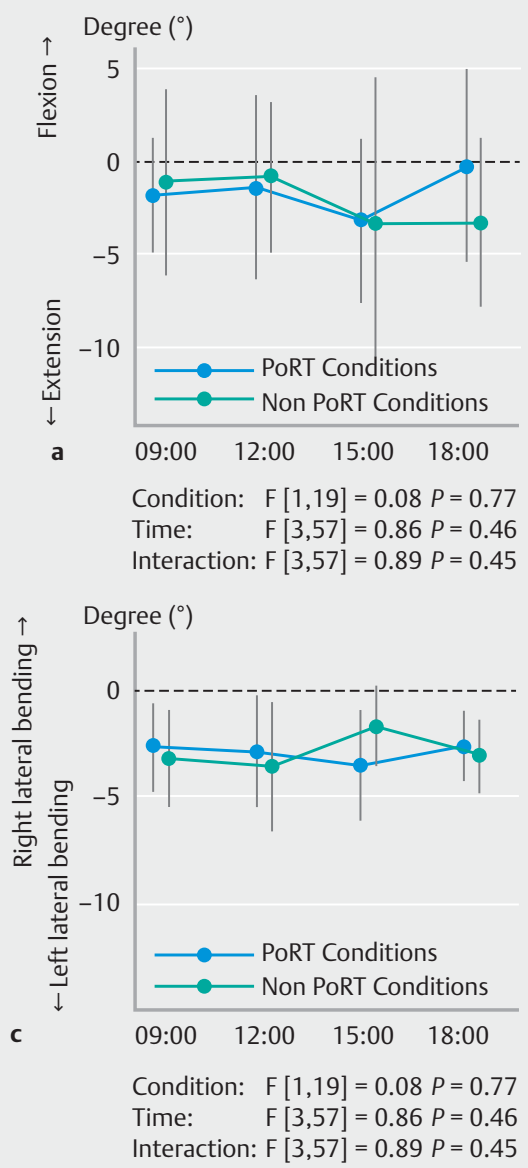
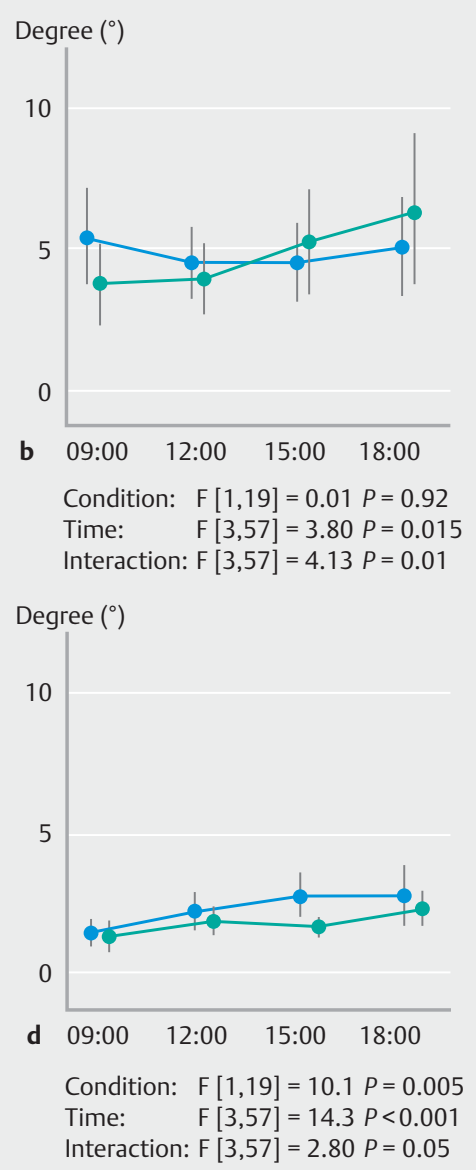

- Fig. 4 Trunk flexion angle: difference between the PoRT and non-PoRT conditions during fluoroscopy procedures. a, c Mean angle.

b, d Mean displacement. Error bars represent the standard deviation.

PoRT condition was significantly higher than that in the nonPoRT condition ( $P=0.005$, $>$ Fig. 4 d). Pitch displacement tended to be constant under the PoRT condition but increased with time under the non-PoRT condition (condition $\times$ time interaction: $P=0.01$, Fig.4b). The main effects of condition and time, and the interaction effect, were statistically significant with respect to roll displacement ( $\triangleright$ Fig. $4 d$ ).

\section{Discussion}

PoRT performed during fluoroscopic endoscopic treatment reduced muscular discomfort in the neck/shoulders, lower back, and lower limbs, and helped keep the head angle at the ideal neutral position of $\sim 0^{\circ}$. However, it had no effect on trunk flexion or the lateral bending posture ( $\mathbf{F i g . 3 a , ~} \mathbf{F i g . 3 c}$ ). On the other hand, only the trunk roll was higher under the PoRT condition than under the non-PoRT condition. Lateral bending during procedures may be more fatiguing than maintaining a neutral trunk angle. Postural dynamics and shifts are good indicators of discomfort [5-7]. With greater accumulation of fatigue, displacement increases, indicating that the extent of displacement reflects attempts to alleviate discomfort or fatigue. This may explain why PoRT was effective in attenuating the develop- ment of discomfort in the neck/shoulders, lower back, and lower limbs, and promoted the maintenance of good neutral posture. Our simple, no-cost intervention involving control of the work-rest schedule (PoRT) may reduce MSDs in medical workers but cannot completely eliminate discomfort that has accumulated over time.

Endoscopic procedures have become increasingly complex and time-consuming. Higher procedural volumes and more years of endoscopy are associated with higher rates of workrelated injuries [2]. The PoRT is a simple and inexpensive countermeasure that may help prevent MSDs by promoting an effective work-rest schedule. A previous study [8] on visual display terminal operators showed that 15-minute work times followed by micro-breaks increased their speed, accuracy, and performance and reduced MSDs. For endoscopists, micro-breaks alone did not reduce MSDs, but they did so in combination with the PoRT. Our results indicate that the PoRT reduces subjective muscular discomfort and improves posture during the performance of fluoroscopic endoscopy, and thus, may enable more efficient work.

The limitations of our study included the fact that, to test the benefits of PoRT, the participant wore RPPE for 9 hours consecutively each day. In addition, this study was conducted in a 
single center under the management of an occupational health specialist. Further, the daily procedural numbers varied. Our study design was single-arm and within-subject, and hence not a randomized controlled trial (RCT). Blinding was impossible. Thus, our evidence is limited to the intra-rater effects of intervention under unblinded conditions. Large-scale, multicenter RCTs are required to confirm that PoRT alleviates MSDs.

\section{Conclusions}

In conclusion, periodic microbreaks involving PoRT can reduce subjective MSD in endoscopists and personnel assisting fluoroscopic procedures. By applying ergonomic measures such as PoRT, medical workers may be less likely to suffer from MSDs.

\section{Acknowledgements}

The study was supported by the Nitto Foundation (grant no. JOSE202100) and the Japan Science and Technology Agency (JST [grant no.JPMJPF2007]). The funders played no roles in the study design, data collection or analysis, interpretation, or writing of the paper. The authors are indebted to Takuya Kushimachi for collecting the data on muscular discomfort.

\section{Competing interests}

The authors declare that they have no conflict of interest.

\section{References}

[1] O'Sullivan S, Bridge G, Ponich T. Musculoskeletal injuries among ERCP endoscopists in Canada. Can J Gastroenterol 2002; 16: 369-374

[2] Ridtitid W, Coté GA, Leung W et al. Prevalence and risk factors for musculoskeletal injuries related to endoscopy. Gastrointest Endosc 2015; 81: 294-302.e4

[3] Pedrosa MC, Farraye FA, Shergill AK et al. Minimizing occupational hazards in endoscopy: personal protective equipment, radiation safety, and ergonomics. Gastrointest Endosc 2010; 72: 227-235

[4] Shergill AK, McQuaid KR, Rempel D. Ergonomics and GI endoscopy. Gastrointest Endosc 2009; 70: 145-153

[5] Ebara T, Kubo T, Inoue T et al. Effects of adjustable sit-stand VDT workstations on workers' musculoskeletal discomfort, alertness and performance. Ind Health 2008; 46: 497-505

[6] Liao MH, Drury CG. Posture, discomfort and performance in a VDT task. Ergonomics 2000; 43: 345-359

[7] Takanishi T, Ebara T, Murasaki Gl et al. Interactive model of subsidiary behaviors, work performance and autonomic nerve activity during visual display terminal work. J Occup Health 2010; 52: 39-47

[8] Balci R, Aghazadeh F. The effect of work-rest schedules and type of task on the discomfort and performance of VDT users. Ergonomics 2003; 46: 455-465 